\title{
The possibility of information loss in transcribing genetic sequence of DNA into proteins due to acceleration of Universe
}

\author{
Alireza Sepehri ${ }^{1}$, Somayyeh Shoorvazi ${ }^{2}$ \\ ${ }^{1}$ Faculty of Physics, Shahid Bahonar University, P.O. Box 76175, Kerman, Iran \\ ${ }^{2}$ Islamic Azad University, Neyshabur branch, Neyshabur, Iran
}

Email address:

A.Sepehri14@gmail.com (A. Sepehri), S.Shoorvazi14@gmail.com (S. Shoorvazi)

To cite this article:

Alireza Sepehri, Somayyeh Shoorvazi. The Possibility of Information Loss in Transcribing Genetic Sequence of DNA into Proteins due to Acceleration of Universe. American Journal of Bioscience and Bioengineering. Vol. 1, No. 2, 2013, pp. 38-42.

doi: $10.11648 /$ j.bio.20130102.12

\begin{abstract}
One of the most exciting subjects in science is the possibility of information loss in transcribing genetic sequence of DNA into proteins due to acceleration of Universe. This research is the first attempt to investigate this subject. First, the probability that DNA is produced due to acceleration of Universe is obtained. It is found that as the Unruh temperature increases, the effect ofacceleration on the production of DNA becomes systematically more effective, because effect at higher temperatures, the energy that is supplied to keep Universe undergoing acceleration becomes more and consequently more DNAs are produced. Then the average number of binding proteins to DNA in two regions of Rindler space of Universe is calculated. The effect of proteins produced in region II doesn't observe in accelerating Universe. One observer in acceleratingUniverse is unable to access information about the whole of space-time since, from his perspective, a communication horizon appears. This can result in a loss of information and a corresponding degradation ofprotein binding to DNA. This information loss disrupts the cellular dynamics and can lead to serious defects, including cancer.Next the averge number of extra base pairs that are produced in DNA structure by the acceleration of Universe is derived. These extra base pairs cause to be stored more information in DNA.These information can be transcribed of DNA into proteins and lead to dangerous activity in Human body.Finally,the probability for substituting AT pair by CG pair in DNA structure is calculated. It's concluded that with increasing the Unruh temperature, more AT base pairs may be substituted by CG pairs and the probability for generating cancer due to this point mutation is increased.
\end{abstract}

Keywords: Acceleration Of Universe, DNA, Information Loss

\section{Introduction}

Recent observational data with high precision suggest that our Universe is currently in an accelerating phase [1,2]. They are consistent with the presence of a nonzero cosmological constant or quantum vacuum energy.According the Unruh idea, there is a similar effect in flat space for a detector undergoing the acceleration of Universe to a detector near event horizon of black hole. Following Hawking's discovery that asymptotically-flat stationary black holes emit thermal radiation: the detector in accelerating Universe appears to be in a heat bath at the Unruh temperature $[3,4]$.One of the most important detectors in Universe is the DNA that information is transcribed from it into proteins.It seems that DNA detects extra proteins due to Unruh temperature of Universe.

One of the most exciting subjects in science is the possibility of information loss in transcribing genetic sequence of DNA into proteins due to acceleration of Universe. It is well known that practically all genetic information in living systems is contained in the deoxyribonucleic acid (DNA) sequence. The DNA is packaged into chromosome which is localized in the nucleus ofthe eukaryotic cells. The famous double-helix structure of DNA is made of two strands that carry organic bases that are bound in pairs by hydrogen bonds, keeping the two strands together.There are 4 types of bases, called A, T, G, C, but only AT and GC pairs are partof the structure of DNA. The AT pairs are bound by two hydrogen bonds, while the stronger GC pairs are bound by three hydrogen bonds[5]. The passage of its gene information to protein, called gene expression, performs by the 
messenger ribonucleic acid (mRNA), which is usually a single polynucleotide chain. RNA molecules carry the nucleotide bases - A, U, C, G-with U very similar in chemical structure to T. A-U pairing is as strong as A-T pairing[6]

In this research we investigate the effects of acceleration of Universe on DNAstructures. The outline of the paper is as follows. In section II we discuss the information loss in DNA due to acceleration of Universe.In section III we discuss the production of extra base pairs in DNA structure due to acceleration of Universe.In section IV we obtain the probability for substituting AT pair by CG pair in DNA structure that leads to generation of cancer. The last section is devoted to summary.

\section{The Information Loss in DNA due to Acceleration of Universe}

TheRindler coordinates are appropriate for describing the viewpoint ofthe Universe moving with acceleration. The world lines ofaccelerated Universe in the Minkowski coordinates correspond to hyperbolae in the left (region I) and right (region II) of the origin which are bounded by light-like asymptotes constituting the Rindler horizon, so two Rindler regions are causally disconnected from each other(see figure1.) $[3,4]$. The Universe thatundergoes the acceleration remains constrained to either Rindler region I or II and has no access to the other sector.

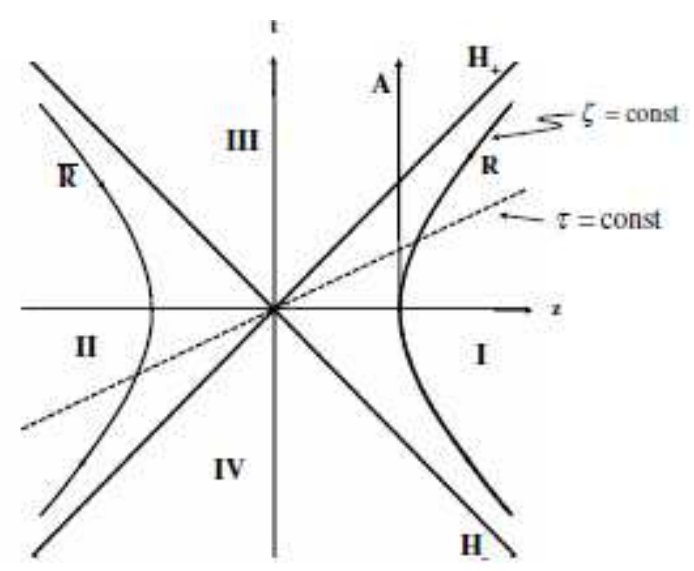

Figure 1. Spacetime diagram, showing the trajectories of an inertial and an accelerated observer [4].

Unruh showed that the accelerated observers in regions I and II detect a thermal distribution of particles at Unruh temperature. This temperature is given by[4]:

$$
T_{U}=\frac{\hbar a}{2 \pi k_{B} c}
$$

where $\mathrm{a}$ is the acceleration of Universe.At this temperature ,DNA maybe detect extra proteins as a signature of the acceleration of Universe.Using the mechanism in ref[7],we consider the protein production near DNA.

The $\beta$ generating Hamiltonian for protein production near DNAdue to accelerating Universe is given by:

$$
\begin{aligned}
& H_{D N A}=\sum_{i=1}^{N} H_{D N A, i}= \\
& \sum_{i=1}^{N}\left(n_{D N A, I}+n_{D N A, I I}\right) \frac{M_{D N A}}{N}+E_{p}\left(n_{I, i}+n_{I I, i}\right)
\end{aligned}
$$

In this Hamiltonian MDNA is the DNA mass, nDNA,I/II indicates whether DNA is produced due to acceleration of Universe $(=1)$ or not $(=0)$ in regions I and II respectively.The variables $\mathrm{n}_{\mathrm{I}, \mathrm{i}}$ and $\mathrm{n}_{\mathrm{II}, \mathrm{i}}$ indicate whether $(=1)$ or not $(=$ 0 ) a protein is bound to site $\mathrm{i}$ of DNAin the regions I and II, respectively. The variable $\mathrm{N}$ is the number of sites that proteins bind to them, $E_{p}$ is the protein energy that causes to change in free energy upon binding a protein to a DNA site. We define $\beta$ as following:

$$
\beta=\frac{1}{k T_{U}}
$$

Tocalculate the probability of a DNA is produced in region I or region II due to acceleration of Universe,we express the partition function as:

$$
\begin{aligned}
& Z_{D N A}=\sum_{n_{D N A, I}=0}^{1} \sum_{n_{D N A, I}=0}^{1} \prod_{i=1}^{N} Z_{i} \\
& Z_{i}=\sum_{n_{I I, i}=0}^{1} \sum_{n_{I, i}=0}^{1} e^{-\beta H_{D N A, i}} \\
& =e^{-\left(n_{D N A, I}+n_{D N A, I}\right) \frac{M_{D N A}}{N k T_{U}}}+ \\
& e^{-\left(\left(n_{D N A, I}+n_{D N A, I I}\right) \frac{M_{D N A}}{N k T_{U}}+2 \frac{E_{p}}{k T_{U}}\right)}+ \\
& 2 e^{-\left(\left(n_{D N A, I}+n_{D N A, I}\right) \frac{M_{D N A}}{N k T_{U}}+\frac{E_{p}}{k T_{U}}\right)}
\end{aligned}
$$

which leads to

$$
\begin{aligned}
& Z_{D N A} \approx\left(e^{-2 \frac{M_{D N A}}{N k T_{U}}}+e^{-\left(2 \frac{M_{D N A}}{N k T_{U}}+2 \frac{E_{p}}{k T_{U}}\right)}\right. \\
& \left.+2 e^{-\left(2 \frac{M_{D N A}}{N k T_{U}}+\frac{E_{p}}{k T_{U}}\right)}\right)^{N}+2\left(e^{-\frac{M_{D N A}}{N k T_{U}}}+e^{-\left(\frac{M_{D N A}}{N k T_{U}}+2 \frac{E_{p}}{k T_{U}}\right)}\right. \\
& \left.+2 e^{-\left(\frac{M_{D N A}}{N k T_{U}}+\frac{E_{p}}{k T_{U}}\right)}\right)^{N}+\left(1+e^{-2 \frac{E_{p}}{k T_{U}}}+2 e^{\frac{E_{p}}{k T_{U}}}\right)^{N}
\end{aligned}
$$

The probability of a DNA is produced due toacceleration of Universein regions I and II is given by the average value of nDNA,I/II. After some mathematical manipulations, we obtain:

$$
\left\langle n_{D N A, I / I I}\right\rangle=-\frac{1}{N \beta} \frac{\partial}{\partial M_{D N A}} \ln Z_{D N A}=\frac{1}{1+F}
$$




$$
\begin{aligned}
& F=\frac{\left(1+e^{-2 \frac{E_{p}}{k T_{U}}}+2 e^{\frac{E_{p}}{k T_{U}}}\right)^{N}}{\left(e^{-2 \frac{M_{D N A}}{N k T_{U}}}+e^{-\left(2 \frac{M_{D N A}}{N k T_{U}}+2 \frac{E_{p}}{k T_{U}}\right)}+2 e^{-\left(2 \frac{M_{D N A}}{N k T_{U}}+\frac{E_{p}}{k T_{U}}\right)}\right)^{N}} \\
& +\left(e^{-\frac{M_{D N A}}{N k T_{U}}}+e^{-\left(\frac{M_{D N A}}{N k T_{U}}+2 \frac{E_{p}}{T_{s}}\right)}+2 e^{-\left(\frac{M_{D N A}}{N k T_{U}}+\frac{E_{p}}{k T_{U}}\right)}\right)^{N}
\end{aligned}
$$

This expression for nDNA,I/IIindicates that, there is the potential for a sharp transition between two states: the DNAis produced due to acceleration ifF $<1$ and isn't produced ifF $>1$. It is found that as the Unruh temperature increases, the effect ofacceleration on the production of DNA becomes systematically more effective, because at higher temperatures ,the energy that is supplied to keep Universe undergoing acceleration becomes more and consequentlymore DNAs is produced. On the other hand at lower temperature, the DNA will not be produced. This leads to a decrease in the probability for DNA production due to acceleration of Universe. We can obtain the average number of binding proteins to DNA in regions I and II as following:

$$
\begin{gathered}
\left\langle n_{I, i / I I, i}\right\rangle=-\frac{1}{N \beta} \frac{\partial}{\partial E_{p}} \ln Z_{D N A}= \\
2\left(e^{-2 \frac{E_{p}}{k T_{U}}}+e^{-\frac{E_{p}}{k T_{U}}}\right)\left(e^{-\frac{(N-1) M_{D N A}}{N k T_{U}}}+1\right)\left(\left(e^{-\frac{M_{D N A}}{N k T_{U}}}+e^{-\left(\frac{M_{D N A}}{N k T_{U}}+2 \frac{E_{p}}{k T_{U}}\right)}\right.\right. \\
\left.+2 e^{-\left(\frac{M_{D N A}}{N k T_{U}}+\frac{E_{p}}{k T_{U}}\right)}\right)^{N-1} \\
+\left(1+e^{-2 \frac{E_{p}}{k T_{U}}}+2 e^{\left.\left.\frac{E_{p}}{k T_{U}}\right)^{N-1}\right)}\right. \\
\left(e^{-2 \frac{M_{D N A}}{N k T_{U}}}+e^{-\left(2 \frac{M_{D N A}}{N k T_{U}}+2 \frac{E_{p}}{k T_{U}}\right)}+2 e^{-\left(2 \frac{M_{D N A}}{N k T_{U}}+\frac{E_{p}}{k T_{U}}\right)}\right)^{N}+ \\
2\left(e^{-\frac{M_{D N A}}{N k T_{U}}}+e^{-\left(\frac{M_{D N A}}{N k T_{U}}+2 \frac{E_{p}}{k T_{U}}\right)}+2 e^{-\left(\frac{M_{S B}}{N k T_{U}}+\frac{E_{p}}{k T_{U}}\right)}\right)^{N} \\
+\left(1+e^{-2 \frac{E_{p}}{k T_{U}}}+2 e^{\frac{E_{p}}{k T_{U}}}\right)^{N} \\
\left\langle n_{I / I I}\right\rangle=N\left\langle n_{I, i / I I, i}\right\rangle
\end{gathered}
$$

This average number depends on the DNA mass.As themass of DNA is very high, the DNA can be source for protein binding.Infact there can be an enormous amount of proteins binding to DNA due to acceleration. The detection of these proteins by DNA,can be a signature of acceleration of Universe.

The effect of proteins produced in region II doesn't observe in accelerating Universe.Oneobserver in acceleratingUniverse is unable to access information about the whole of space-time since, from his perspective, a communication horizon appears. This can result in a loss of information and a corresponding degradation ofprotein binding to DNA.

\section{due to Acceleration of Universe in DNA}

There is a difference between predicted and detected number ofbase pairs in DNA.Because some extra base pairs are produced due to acceleration of Universe. The generating Hamiltonian for base pair production in accelerating Universe is given by:

$$
\begin{aligned}
& H_{\text {base pair }}=n_{\text {a-base pair }, I} E_{\text {base pair-DNA }} \\
& +n_{\text {a-base pair,II }} E_{\text {base pair-DNA }}
\end{aligned}
$$

In this Hamiltonian, Ebase pair-DNA is the correlation energy that correlates base pair to DNA and na-base pair,I and na-base pair,II indicate the number ofbase pairs that are produced in the regions I and II due to acceleration of Universe, respectively.To count the thermal distribution for base pairs we write the partition function as a function of Unruh temperature as

$$
\begin{aligned}
& Z_{\text {base pair }}=\sum_{n_{I, i}=0}^{1} \sum_{n_{I, i}=0}^{1} e^{-\frac{H_{\text {base pair }}}{k T_{U}}}= \\
& 2 e^{-\frac{E_{\text {base }} \text { pair-DNA }}{k T_{U}}}+e^{-\frac{2 E_{\text {base pair-DNA }}}{k T_{U}}}+1
\end{aligned}
$$

The averge number ofbase pairs that are produced by the acceleration of Universe is given by the average value of na-base pair.

$$
\begin{aligned}
& \left\langle n_{\text {a-base pair }, I / I I}\right\rangle= \\
& -\frac{k T_{U}}{2} \frac{\partial}{\partial E_{\text {base pair-DNA }}} \ln Z_{\text {base pair }} \\
& =\frac{e^{-\frac{E_{\text {base pair-DNA }}}{k T_{U}}}}{e^{-\frac{E_{\text {base pair-DNA }}}{k T_{U}}}+1}
\end{aligned}
$$

As the Unruh temperature of Universe is very high, there can be an amount of base pair production in DNA structure. These extra base pairs cause to be stored more information in DNA.These information can be transcribed of DNA into proteins and lead to dangerous activity in Human body.

\section{The Probability for Generating Can- cer due to Acceleration of Universe}

Recently,it has been shown that most of cancerous mutations in DNA are point mutation in which CG pair is substituted by AT pair[8,9]. We can write the generating Hamiltonian for this mutation as following:

\section{The Production of Extra Base Pairs}




$$
\begin{aligned}
& H_{\text {cancer }}=\sum_{i=1}^{N} n_{A T-D N A, i} E_{A T-D N A}+ \\
& n_{C G-D N A, i} E_{C G-D N A}+n_{C G-A T} \frac{E_{A T-C G}}{N}
\end{aligned}
$$

In this Hamiltonian nCG-AT indicates whether CG pair is substitute by AT pair $(=1)$ or not $(=0)$. The variables nAT-DNA,i and nCG-DNA,i indicate whether $(=1)$ or not $(=0)$ AT or CG pairis located in site i of DNA, respectively. The variable $\mathrm{N}$ is the number of sites that base pairs are correlated to DNA, EAT-DNA and ECG-DNA are the energies that are supplied to keep AT and CG pairs entangled to DNA, ECG-AT is the energy that causes to change in free energy upon substituting CG by AT.

Tocalculate the probability of a AT being substituted by $\mathrm{CG}$,we express the partition function as:

$$
\begin{aligned}
& Z_{2}=\sum_{n_{A}=0}^{1} \prod_{i=1}^{N} Z_{i} \\
& Z_{i}=\sum_{n_{I I,}=0}^{1} \sum_{n_{I, i}=0}^{1} e^{-\beta H_{2, i}}= \\
& e^{-\frac{n_{C G-A T} E_{A T-C G}}{N K T_{U}}}+e^{-\left(\frac{n_{C G-A T} E_{A T-C G}}{N K T_{U}}+\frac{E_{C G-D N A}}{K T_{U}}+\frac{E_{A T-D N A}}{K T_{U}}\right)} \\
& +e^{-\left(\frac{E_{A T-C G}}{N K T_{U}}+\frac{E_{A T-D N A}}{K T_{U}}\right)}+e^{-\left(\frac{n_{C G-A T} E_{A T-C G}}{N K T_{U}}+\frac{E_{C G-D N A}}{K T_{U}}\right)}
\end{aligned}
$$

which leads to

$$
\begin{aligned}
& Z_{2} \approx\left(e^{-\frac{E_{A T-C G}}{N K T_{U}}}+e^{-\left(\frac{E_{A T-C G}}{N K T_{U}}+\frac{E_{C G-D N A}}{K T_{U}}+\frac{E_{A T-D N A}}{K T_{U}}\right)}\right. \\
& \left.+e^{-\left(\frac{E_{A T-C G}}{N K T_{U}}+\frac{E_{A T-D N A}}{K T_{U}}\right)}+e^{-\left(\frac{E_{A T-C G}}{N K T_{U}}+\frac{E_{C G-D N A}}{K T_{U}}\right)}\right)^{N} \\
& +\left(1+e^{-\left(\frac{E_{C G-D N A}}{K T_{U}}+\frac{E_{A T-D N A}}{K T_{U}}\right)}\right. \\
& \left.+e^{-\left(\frac{E_{A T-D N A}}{K T_{U}}\right)}+e^{-\left(\frac{E_{C G-D N A}}{K T_{U}}\right)}\right)^{N}
\end{aligned}
$$

The probability of a AT being substituted by $\mathrm{CG}$ is given by the average value of nCG-AT. After some mathematical manipulations, we obtain

$$
\begin{gathered}
\left\langle n_{C G-A T}\right\rangle=-\frac{1}{\beta} \frac{\partial}{\partial E_{A T-C G}} \ln Z_{2}=\frac{1}{1+F^{N}} \\
1+e^{-\left(\frac{E_{C G-D N A}}{K T_{U}}+\frac{E_{A T-D N A}}{K T_{U}}\right)} \\
F=\frac{+e^{-\left(\frac{E_{A T-D N A}}{K T_{U}}\right)}+e^{-\left(\frac{E_{C G-D N A}}{K T_{U}}\right)}}{e^{-\frac{E_{A T-C G}}{N K T_{U}}}+e^{-\left(\frac{E_{A T-C G}}{N K T_{U}}+\frac{E_{C G-D N A}}{K T_{U}}+\frac{E_{A T-D N A}}{K T_{U}}\right)}} \\
+e^{-\left(\frac{E_{A T-C G}}{N K T_{U}}+\frac{E_{A T-D N A}}{K T_{U}}\right)}+e^{-\left(\frac{E_{A T-C G}}{N K T_{U}}+\frac{E_{C G-D N A}}{K T_{U}}\right)}
\end{gathered}
$$

This expression for nCG-ATindicates that, there is the potential for a sharp transition between two states: the AT is substituted by $\mathrm{CG}$ ifF $<1$ and isn't substitutedifF $>1$. This average number depends on the Unruh temperature of accelerating Universe.With increasing the Unruh temperature, more AT base pairs may be substituted by CG pairs and the probability for generating cancer is increased.

\section{Summary}

In this research, first we obtain the probability of a DNA is produced due toacceleration of Universe. It is found that as the Unruh temperature increases, the effect ofacceleration on the production of DNA becomes systematically more effective, because at higher temperatures, the energy that is supplied to keep Universe undergoing acceleration becomes more and consequentlymore DNAs is produced.Then we calculate the average number of binding proteins to DNA in two regions of Rindler space. The effect of proteins produced in region II doesn't observe in accelerating Universe.Oneobserver in acceleratingUniverse is unable to access information about the whole of space-time since, from his perspective, a communication horizon appears. This can result in a loss of information and a corresponding degradation ofprotein binding to DNA. This information loss disrupts the cellular dynamics and can lead to serious defects, including cancer.Next we derive the average number of extra base pairs that are produced in DNA structure by the acceleration of Universe. These extra base pairs cause to be stored more information in DNA. Finally,we calculate the probability for substituting AT pair by CG pair in DNA structure. With increasing the Unruh temperature, more AT base pairs may be substituted by CG pairs and the probability for generating cancer is increased.

\section{Acknowledgments}

we would like to thank of A. Abitaleb and G.Majid for their lectures about the creation of the Universe and quantum biology that gave us new insight into these subjects.

\section{References}

[1] S. Perlmutter et al., Nature 391 (1998) 51

[2] D. N. Spergel et al.,Astrophys. J. Suppl. 148, 175 (2003)

[3] I. Fuentes-Schuller and R. B. Mann, Phys. Rev. Lett. 95, 120404 (2005).

[4] P. M. Alsing, I. Fuentes-Schuller, R. B. Mann, and T. E. Tessier, Phys. Rev.A74, 032326 (2006).

[5] Michel Peyrard, Santiago Cuesta-Lopez, Dimitar Angelov, Journal of Physics: Condensed Matter 3, 21 (2008) 034103$1-13$

[6] Branko Dragovich, Alexandra Dragovich, p-Adic Numbers,Ultrametric Analysis and Applications 1 (2009) 34-41 
[7] Jose M. G. Vilar and Leonor Saiz,Phys. Rev. Lett. 96, 238103 (2006)

[8] C. J. Sherr, Cell 234, 116 (2004).
[9] A. Petitjean et al., Hum. Mutat. 28, 622 (2007), Chi-Tin Shih, Stephan Roche, Rudolf A. Römer, Phys. Rev. Lett. $100,018105(2008)$ 